\title{
Qatar Exoplanet Survey : Qatar-3b, Qatar-4b, and Qatar-5b
}

\author{
Khalid Alsubai $^{1}$, Dimitris Mislis ${ }^{1}$, Zlatan I. Tsvetanov ${ }^{1}$, David W. Latham ${ }^{2}$, Allyson Bieryla ${ }^{2}$, Lars A. Buchhave ${ }^{3}$, \\ Gilbert A. Esquerdo ${ }^{2}$, D. M. Bramich ${ }^{1}$, Stylianos Pyrzas ${ }^{1}$, Nicolas P. E. Vilchez ${ }^{1}$, Luigi Mancini ${ }^{4,5}$, John Southworth ${ }^{6}$, \\ Daniel F. Evans ${ }^{6}$, Thomas Henning ${ }^{4}$, and Simona Ciceri ${ }^{4,7}$ \\ ${ }^{1}$ Qatar Environment and Energy Research Institute (QEERI), HBKU, Qatar Foundation, P.O. Box 5825, Doha, Qatar; kalsubai@qf.org.qa \\ ${ }^{2}$ Harvard-Smithsonian Center for Astrophysics, 60 Garden Street, Cambridge, MA 02138, USA \\ ${ }^{3}$ Centre for Star and Planet Formation, Natural History Museum of Denmark \& Niels Bohr Institute, \\ University of Copenhagen, Øster Voldgade 5-7, DK-1350 Copenhagen K, Denmark \\ ${ }^{4}$ Max Planck Institute for Astronomy, Königstuhl 17, D-69117 Heidelberg, Germany \\ ${ }^{5}$ INAF-Osservatorio Astrofisico di Torino, via Osservatorio 20, I-10025, Pino Torinese, Italy \\ ${ }^{6}$ Astrophysics Group, Keele University, Staffordshire ST5 5BG, UK \\ ${ }^{7}$ Department of Astronomy, Stockholm University, AlbaNova University Centre, SE-106 91 Stockholm, Sweden \\ Received 2016 December 22; revised 2017 February 19; accepted 2017 February 23; published 2017 April 4
}

\begin{abstract}
We report the discovery of Qatar-3b, Qatar-4b, and Qatar-5b, three new transiting planets identified by the Qatar Exoplanet Survey. The three planets belong to the hot Jupiter family, with orbital periods of $P_{\mathrm{Q} 3 \mathrm{~b}}=2.50792$ days, $P_{\mathrm{Q} 4 \mathrm{~b}}=1.80539$ days, and $P_{\mathrm{Q} 5 \mathrm{~b}}=2.87923$ days. Follow-up spectroscopic observations reveal the masses of the planets to be $M_{\mathrm{Q} 3 \mathrm{~b}}=4.31 \pm 0.47 M_{\mathrm{J}}, M_{\mathrm{Q} 4 \mathrm{~b}}=6.10 \pm 0.54 M_{\mathrm{J}}$, and $M_{\mathrm{Q} 5 \mathrm{~b}}=4.32 \pm 0.18 M_{\mathrm{J}}$, while model fits to the transit light curves yield radii of $R_{\mathrm{Q} 3 \mathrm{~b}}=1.096 \pm 0.14 R_{\mathrm{J}}, \quad R_{\mathrm{Q} 4 \mathrm{~b}}=1.135 \pm 0.11 \quad R_{\mathrm{J}}$, and $R_{\mathrm{Q} 5 \mathrm{~b}}=$ $1.107 \pm 0.064 R_{\mathrm{J}}$. The host stars are low-mass main sequence stars with masses and radii $M_{\mathrm{Q} 3}=$ $1.145 \pm 0.064 M_{\odot}, \quad M_{\mathrm{Q} 4}=0.896 \pm 0.048 M_{\odot}, \quad M_{\mathrm{Q} 5}=1.128 \pm 0.056 M_{\odot} \quad$ and $\quad R_{\mathrm{Q} 3}=1.272 \pm 0.14 R_{\odot}$, $R_{\mathrm{Q} 4}=0.849 \pm 0.063 R_{\odot}$, and $R_{\mathrm{Q} 5}=1.076 \pm 0.051 R_{\odot}$ for Qatar-3, 4, and 5 respectively. The $V$ magnitudes of the three host stars are $V_{\mathrm{Q} 3}=12.88, V_{\mathrm{Q} 4}=13.60$, and $V_{\mathrm{Q} 5}=12.82$. All three new planets can be classified as heavy hot Jupiters $\left(M>4 M_{\mathrm{J}}\right)$.
\end{abstract}

Key words: planetary systems - planets and satellites: gaseous planets

\section{Introduction}

Ground-based surveys for transiting exoplanets continue to be a productive source for finding short period giant planets orbiting relatively bright stars. Many of these discoveries have become primary targets for subsequent studies of exoplanetary atmospheres and other important planetary characteristics with the use of some of the most advanced ground- and space-based telescopes. In addition, these discoveries contribute significantly to a more complete census of hot Jupiters and other close orbiting large planets-the type of planets not present in our solar system - and may provide a key to understanding their origin and more generally the planetary system architecture.

This paper is based on observations collected with the first generation of the Qatar Exoplanet Survey (QES, Alsubai et al. 2013). QES uses two overlapping wide field $135 \mathrm{~mm}$ $(f / 2.0)$ and $200 \mathrm{~mm}(f / 2.0)$ telephoto lenses, along with four $400 \mathrm{~mm}(f / 2.8)$ telephoto lenses, mosaiced to image an $11^{\circ} \times 11^{\circ}$ field on the sky simultaneously at three different pixel scales. The three different pixel scales are 12, 9, and 4 arcsec respectively for the three different types of lenses. With its larger aperture lenses, its higher angular resolution (a result of the longer focal length of the lenses), and the detrending algorithms, QES is able to reach $1 \%$ photometric accuracy up to $13.5-14.0 \mathrm{mag}$.

In this paper we present the discovery of three new hot Jupiters from QES, namely Qatar-3b, Qatar- $4 b$, and Qatar-5b. The paper is organized as follows: in Section 2 we present the survey photometry and describe the follow-up spectroscopy and photometry used to confirm the planetary nature of the transits. In Section 3 we present the global system solutions using simultaneous fits to the available RV and follow-up photometric light curves with the stellar parameters determined from the combined spectra, while in Section 4 we summarise our results.

\section{Observations}

\subsection{Discovery Photometry}

Observations for the discovery photometry were collected at the QES station in New Mexico, USA. QES utilizes FLI ProLine PL6801 cameras, with KAF-1680E 4k $\times$ 4k detectors. Exposure times were $60 \mathrm{~s}$, for each of the four CCDs attached to the $400 \mathrm{~mm}$ lenses; $45 \mathrm{~s}$, for the CCD equipped with the $200 \mathrm{~mm}$ lens; and $30 \mathrm{~s}$, for the CCD equipped with the $135 \mathrm{~mm}$ lens.

The survey data were reduced with the QES-pipeline, which performs bias-correction, dark-current subtraction and flatfielding in the standard fashion, while photometric measurements are extracted using the image subtraction algorithm by Bramich (2008); a more detailed description of the pipeline is given in Alsubai et al. (2013).

The output light curves were ingested into the QES archive and subsequently subjected to a combination of the Trend Filtering Algorithm (Kovács et al. 2005) and the SysRem algorithm (Tamuz et al. 2005), to model and remove systematic patterns of correlated noise. Transit-like events for all three stars were identified using the Box Least Square algorithm (BLS) of Kovács et al. (2002), during a candidates' search on the archive light curves following the procedure described in Collier Cameron et al. (2006). We note that the initial candidate selection is an automatic procedure, but the final candidate vetting is done by eye. The BLS algorithm provided tentative 


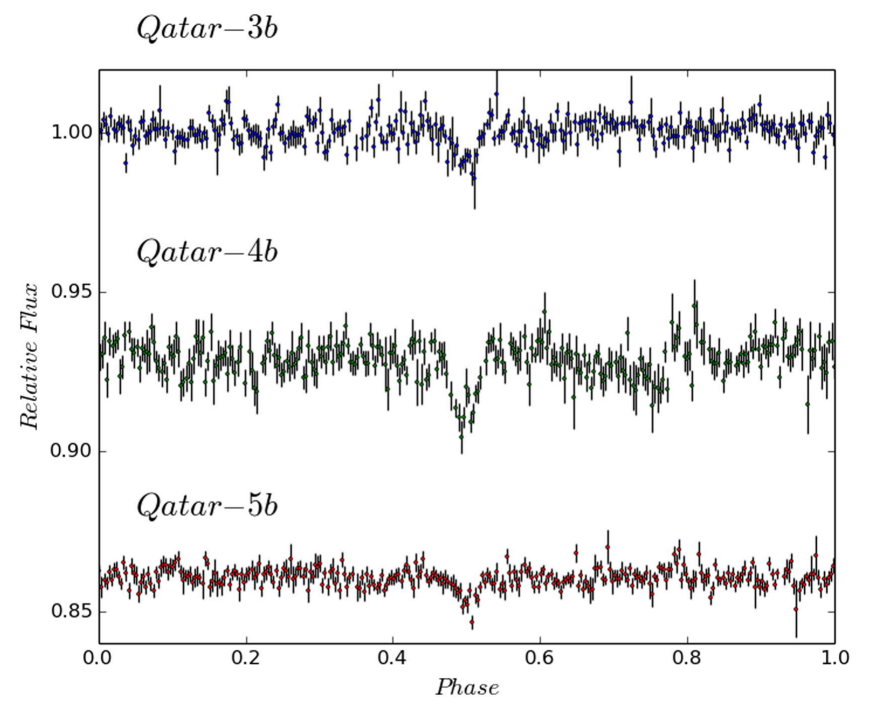

Figure 1. The discovery light curves phase folded with the BLS estimated periods, as they appear in the QES archive, for Qatar-3b (top, in blue), Qatar-4b (middle, in green), and Qatar-5b (bottom, in red). For clarity, all light curves have been binned using a mean filter by a factor of 35, while those of Qatar- $4 \mathrm{~b}$ and Qatar-5b have been shifted downwards.

ephemerides which were used to phase-fold the discovery light curves shown in Figure 1.

The discovery light curve of Qatar-3b contains data points from 11,228 frames, spanning a period from 2012 October to 2015 January; that of Qatar-4b contains data points from 8950 frames, with a time-span from 2012 September to 2014 November, and that of Qatar-5b contains 18,957 data points, with a time-span from 2012 September to 2014 December.

\subsection{The Host Stars}

Qatar-3b's host is a $V=12.88 \mathrm{mag}(B=13.13 \mathrm{mag})$ star (UCAC3 ID: 253-304972, henceforth designated Qatar-3) of spectral type very close to G0V. The host of Qatar-4b is a $V=13.60 \mathrm{mag}(B=14.69 \mathrm{mag})$, early-K type star (UCAC3 ID: 269-003518, henceforth designated Qatar-4), and, similarly, the host of Qatar-5b is a $V=12.82 \mathrm{mag}(B=13.00 \mathrm{mag})$ star (UCAC3 ID: 265-004681, henceforth designated Qatar-5) of spectral type close to G2V. The basic observational characteristics of the three host stars, together with the results from the spectroscopic analysis, are listed in Table 1. We further discuss stellar parameters determined from our followup spectra in Section 3.1. The host star spectral types are estimated from a multi-color fit ( $\mathrm{J}, \mathrm{H}, \mathrm{V}$, and $\mathrm{K}$ band) to the UCAC3 values, using a standard Random-Forest classification algorithm, trained with $\sim 200$ standards with spectral types ranging from early $\mathrm{A}$ to late $\mathrm{M}$.

\subsection{Follow-up Spectroscopy}

Follow-up spectroscopic observations of all three candidates were obtained with the Tillinghast Reflector Echelle Spectrograph (TRES) on the $1.5 \mathrm{~m}$ Tillinghast Reflector at the Fred L. Whipple Observatory on Mount Hopkins, Arizona. Similarly to our campaigns for all QES candidates we used TRES with the medium fiber, which yields a resolving power of $R \sim 44,000$, corresponding to a velocity resolution element of $6.8 \mathrm{~km} \mathrm{~s}^{-1}$ FWHM. The spectra were extracted using version 2.55 of the code described in Buchhave et al. (2010). The wavelength calibration for each spectrum was established using
Table 1

Basic Observational and Spectroscopic Parameters of the Host Stars

\begin{tabular}{llll}
\hline \hline Parameters & Qatar-3 & Qatar-4 & Qatar-5 \\
\hline ID (UCAC3) & $253-304972$ & $269-003518$ & $265-004681$ \\
$\alpha_{2000}$ & $23^{\mathrm{h}} 56^{\mathrm{m}} 36.48$ & $00^{\mathrm{h}} 19^{\mathrm{m}} 26.22$ & $00^{\mathrm{s}} 28^{\mathrm{m}} 12^{\mathrm{s}} .94$ \\
$\delta_{2000}$ & $+36^{\circ} 12^{\prime} 46^{\prime \prime} 6$ & $+44^{\circ} 01^{\prime} 39^{\prime \prime} 4$ & $+42^{\circ} 03^{\prime} 400^{\prime \prime} 9$ \\
$V(\mathrm{mag})$ & 12.88 & 13.60 & 12.82 \\
$B(\mathrm{mag})$ & 13.13 & 14.69 & 13.00 \\
$J(\mathrm{mag})$ & 11.60 & 13.61 & 11.35 \\
Spectral Types & $\mathrm{G} 0 \mathrm{~V}$ & $\mathrm{~K} 1 \mathrm{~V}$ & $\mathrm{G} 2 \mathrm{~V}$ \\
& & & $5746 \pm 50$ \\
$T_{\text {eff }}(\mathrm{K})$ & $6009 \pm 52$ & $5218 \pm 51$ & $4.43 \pm 0.10$ \\
$\log g(\mathrm{cgs})$ & $4.29 \pm 0.10$ & $4.59 \pm 0.10$ & $0.38 \pm 0.08$ \\
{$[M / H]$} & $-0.04 \pm 0.08$ & $0.10 \pm 0.08$ & $4.5 \pm 0.5$ \\
$v \sin i\left(\mathrm{~km} \mathrm{~s}^{-1}\right)$ & $10.2 \pm 0.5$ & $7.1 \pm 0.5$ & \\
\hline
\end{tabular}

exposures of a thorium-argon hollow-cathode lamp illuminating the science fiber, obtained immediately before and after each observation of the star.

For Qatar-3, a total of 27 spectra were obtained between 2015 July 30 (UT) and 2016 January 03 with a typical exposure time of 30 minutes and an average signal-to-noise ratio per resolution element $(\mathrm{S} / \mathrm{Ne})$ of 29 at the peak of the continuum in the echelle order centered on the $\mathrm{Mg}$ b triplet near $519 \mathrm{~nm}$. For Qatar-4, we obtained 8 usable spectra between 2015 September 23 and 2016 February 09 with mostly 48 minutes exposures and $\langle\mathrm{S} / \mathrm{Ne}\rangle=22$, and for Qatar-5 we obtained a total of 25 usable spectra between 2015 September 27 and 2015 December 08 with mostly 25 minutes exposures and $\langle\mathrm{S} / \mathrm{Ne}\rangle=29$.

Relative radial velocities (RVs) were derived by crosscorrelating each observed spectrum against the strongest exposure of the same star, order by order for a set of echelle orders selected to have good $\mathrm{S} / \mathrm{Ne}$ and minimal contamination by telluric lines introduced by the Earth's atmosphere. These RVs are reported in Tables 2-4 and the time units are in Barycentric Julian Date in Barycentric Dynamical time $\left(\mathrm{BJD}_{\mathrm{TDB}}\right)$. The observation that was used for the template spectrum for each star has, by definition, an RV of $0.00 \mathrm{~km} \mathrm{~s}^{-1}$. We define the error on the template RV as the smallest error of all the other errors. We also derived values for the line profile bisector spans (BSs, lower panel in Figures 2-4), to check for astrophysical phenomena other than orbital motion that might produce a periodic signal in the RVs with the same period as the photometric ephemerides for the transits. The procedures used to determine RVs and BSs are outlined in Buchhave et al. (2010).

To illustrate the quality of the orbital solutions provided by our relative RVs, we fit circular orbits with the epoch and period set to the final ephemerides values from the global analysis. The key parameters for these orbital solutions are reported in Table 5, and the corresponding RV curves and individual observations are plotted in Figures 2-4. Note that the relative gamma velocity is the center-of-mass velocity using the relative velocities.

The values of the correlation coefficient between Bisectors and RVs for Qatar-3b (0.2346) and Qatar-5b (0.153) are low and suggest the correlation is not significant. The value for Qatar-4b is moderate $(0.4485)$, but there are only 10 observations, so the interpretation is inconclusive. We do not calculate the FWHM of the correlation function. As an 
Table 2

Relative RVs and BS Variations for Qatar-3

\begin{tabular}{lcr}
\hline \hline BJD $_{\text {TDB }}$ & RV $\left(\mathrm{m} \mathrm{s}^{-1}\right)$ & \multicolumn{1}{c}{$\mathrm{BS}\left(\mathrm{m} \mathrm{s}^{-1}\right)$} \\
\hline 2457233.9060 & $1314 \pm 49$ & $266 \pm 40$ \\
2457237.8276 & $337 \pm 77$ & $236 \pm 77$ \\
2457263.8334 & $1464 \pm 101$ & $39 \pm 38$ \\
2457271.9555 & $1089 \pm 120$ & $200 \pm 87$ \\
2457273.8826 & $927 \pm 51$ & $6 \pm 54$ \\
2457284.8017 & $732 \pm 102$ & $23 \pm 79$ \\
2457285.9482 & $242 \pm 75$ & $-31 \pm 54$ \\
2457288.7856 & $798 \pm 58$ & $-42 \pm 39$ \\
2457289.8705 & $718 \pm 77$ & $-47 \pm 31$ \\
2457291.6720 & $1042 \pm 77$ & $48 \pm 31$ \\
2457292.7829 & $85 \pm 53$ & $-15 \pm 29$ \\
2457293.7437 & $194 \pm 64$ & $21 \pm 36$ \\
2457294.8549 & $228 \pm 106$ & $-7 \pm 35$ \\
2457295.9123 & $126 \pm 59$ & $-30 \pm 41$ \\
2457296.8432 & $956 \pm 70$ & $7 \pm 38$ \\
2457297.7507 & $19 \pm 70$ & $-14 \pm 28$ \\
2457298.8203 & $647 \pm 62$ & $-31 \pm 21$ \\
2457299.7981 & $713 \pm 113$ & $28 \pm 57$ \\
2457303.8631 & $730 \pm 77$ & $47 \pm 73$ \\
2457304.8158 & $711 \pm 96$ & $-91 \pm 57$ \\
2457315.6195 & $92 \pm 128$ & $-73 \pm 87$ \\
2457318.6972 & $291 \pm 60$ & $-83 \pm 45$ \\
2457328.8461 & $591 \pm 69$ & $-63 \pm 35$ \\
2457332.8579 & $281 \pm 64$ & $-49 \pm 72$ \\
2457351.6699 & $953 \pm 53$ & $-325 \pm 37$ \\
2457357.6141 & $636 \pm 75$ & $-17 \pm 29$ \\
2457390.6129 & $0 \pm 49$ & $2 \pm 37$ \\
& &
\end{tabular}

Table 3

Relative RVs and BS Variations for Qatar-4

\begin{tabular}{lrr}
\hline \hline BJD $_{\text {TDB }}$ & \multicolumn{1}{c}{ RV $\left(\mathrm{m} \mathrm{s}^{-1}\right)$} & \multicolumn{1}{c}{ BS $\left(\mathrm{m} \mathrm{s}^{-1}\right)$} \\
\hline 2457288.86074 & $1878.8 \pm 73.0$ & $3.4 \pm 27.2$ \\
2457296.88246 & $0.0 \pm 59.8$ & $-6.8 \pm 17.3$ \\
2457297.86558 & $1784.5 \pm 51.9$ & $-45.0 \pm 16.2$ \\
2457327.79409 & $-23.4 \pm 63.7$ & $27.3 \pm 24.4$ \\
2457356.60189 & $-33.8 \pm 51.9$ & $-43.0 \pm 23.0$ \\
2457390.66755 & $263.1 \pm 52.4$ & $-43.8 \pm 16.9$ \\
2457409.66062 & $227.7 \pm 83.8$ & $15.4 \pm 32.8$ \\
2457417.61546 & $612.7 \pm 75.8$ & $-3.0 \pm 16.4$ \\
2457653.93140 & $1274.1 \pm 59.8$ & $26.9 \pm 29.6$ \\
2457734.75109 & $2417.3 \pm 47.6$ & $68.6 \pm 17.9$ \\
\hline
\end{tabular}

alternative approach, we run SPC and derive the $v \sin i$ values as a measure of the broadening.

To get the absolute gamma (center-of-mass) velocity for a system where we use the multi-order relative velocities to derive the orbital solution, we have to provide an absolute velocity for the observation that was used for the template when deriving the relative velocities. By definition that observation is assigned a relative velocity of $0.00 \mathrm{~km} \mathrm{~s}^{-1}$. To derive an absolute velocity for that observation, we correlate the $\mathrm{Mg} \mathrm{b}$ order against the template from the CfA library of synthetic templates that gives the highest peak correlation value. Then we add the relative gamma velocity from the orbital solution, and also correct by $-0.61 \mathrm{~km} \mathrm{~s}^{-1}$, mostly because the CfA library does not include the gravitational redshift. This offset has been determined empirically by many observations of IAU RV Standard Stars. We quote an uncertainty in the resulting absolute velocity of $\pm 0.1 \mathrm{~km} \mathrm{~s}^{-1}$,
Table 4

Relative RVs and BS Variations for Qatar-5

\begin{tabular}{lrr}
\hline \hline BJD & $\mathrm{RV}\left(\mathrm{m} \mathrm{s}^{-1}\right)$ & $\mathrm{BS}\left(\mathrm{m} \mathrm{s}^{-1}\right)$ \\
\hline 2457292.7383 & $991 \pm 29$ & $160 \pm 28$ \\
2457296.9362 & $-5 \pm 31$ & $81 \pm 31$ \\
2457298.8466 & $950 \pm 28$ & $34 \pm 17$ \\
2457299.7752 & $95 \pm 38$ & $-17 \pm 24$ \\
2457318.7366 & $1050 \pm 27$ & $-9 \pm 24$ \\
2457327.8283 & $828 \pm 33$ & $5 \pm 28$ \\
2457328.6901 & $-92 \pm 29$ & $-3 \pm 19$ \\
2457329.8154 & $680 \pm 31$ & $-2 \pm 27$ \\
2457332.8336 & $823 \pm 27$ & $-12 \pm 24$ \\
2457345.7245 & $58 \pm 37$ & $1 \pm 21$ \\
2457346.6775 & $210 \pm 27$ & $16 \pm 21$ \\
2457347.6982 & $984 \pm 15$ & $-3 \pm 15$ \\
2457348.6916 & $0 \pm 15$ & $-16 \pm 10$ \\
2457349.6621 & $260 \pm 23$ & $-38 \pm 25$ \\
2457350.6435 & $837 \pm 30$ & $-41 \pm 21$ \\
2457351.6976 & $-235 \pm 20$ & $-28 \pm 11$ \\
2457354.6615 & $-186 \pm 30$ & $-33 \pm 17$ \\
2457355.7121 & $632 \pm 30$ & $-28 \pm 22$ \\
2457356.6458 & $811 \pm 26$ & $5 \pm 15$ \\
2457357.6722 & $-95 \pm 30$ & $2 \pm 21$ \\
2457358.7104 & $741 \pm 25$ & $-24 \pm 21$ \\
2457360.6173 & $-108 \pm 32$ & $1 \pm 28$ \\
2457361.6212 & $775 \pm 28$ & $-48 \pm 19$ \\
2457362.6705 & $464 \pm 24$ & $5 \pm 22$ \\
2457364.6030 & $919 \pm 27$ & $-4 \pm 20$ \\
\hline & &
\end{tabular}

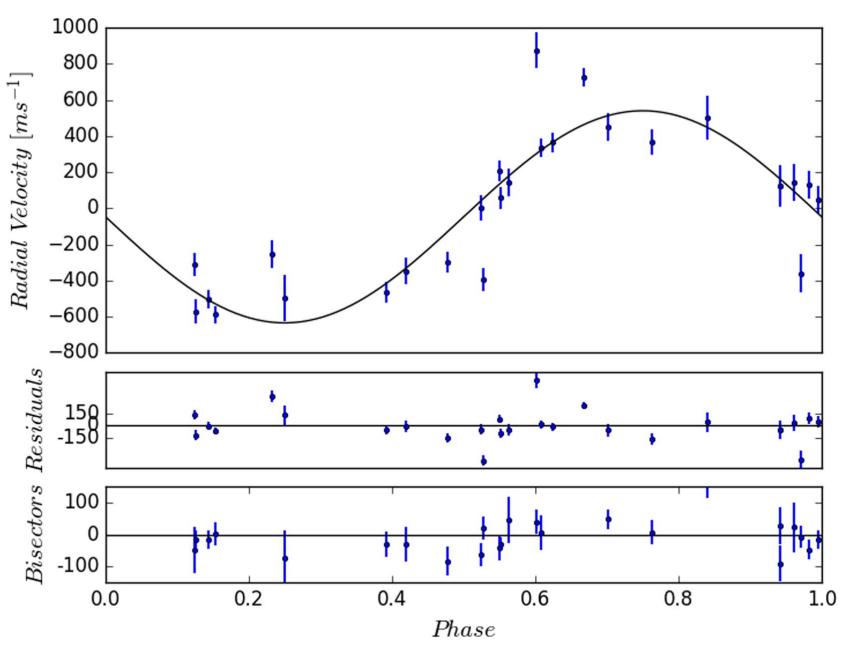

Figure 2. Orbital solution for Qatar-3b, showing the velocity curve and observed velocities.

which is an estimate of the residual systematic errors in the IAU RV Standard Star system.

\subsection{Follow-up Photometry}

Follow-up photometric observations for Qatar-3b and Qatar$4 \mathrm{~b}$ were obtained with the $1.23 \mathrm{~m}$ Zeiss Telescope at the Calar Alto Observatory (CAHA, Spain), using a Cousins-I filter and an exposure time of $60 \mathrm{~s}$ per frame. For all observations, the telescope was defocused and data reduction was carried out using the DEFOT pipeline (Southworth et al. 2009, 2014). Qatar-3b was observed on two occasions, on the 2015 October 6 and 11, while a half-transit of Qatar-4b was observed on the 2015 October 27. Two additional transits of Qatar-4b were observed with the $50 \mathrm{~cm}$ QES Follow-up Telescope (QFT) 


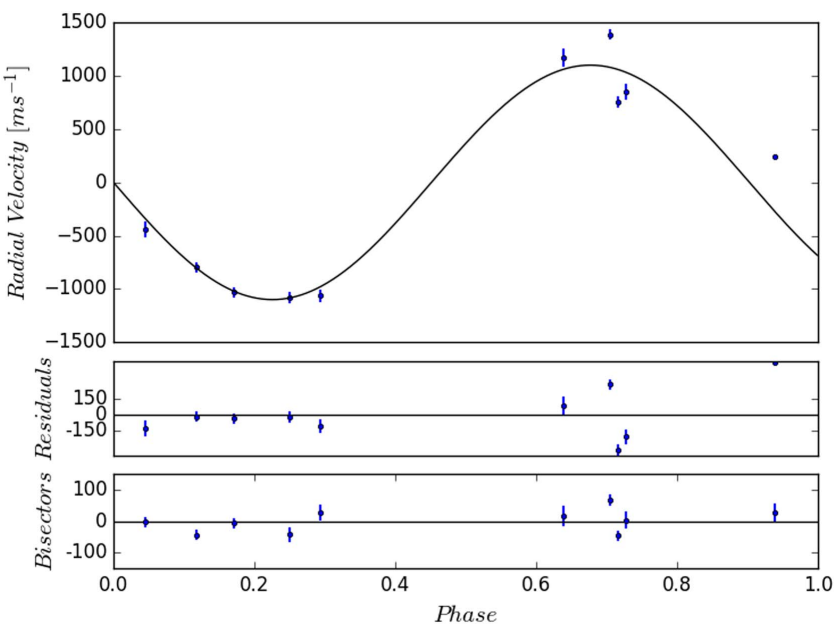

Figure 3. Orbital solution for Qatar- $4 \mathrm{~b}$, showing the velocity curve and observed velocities.

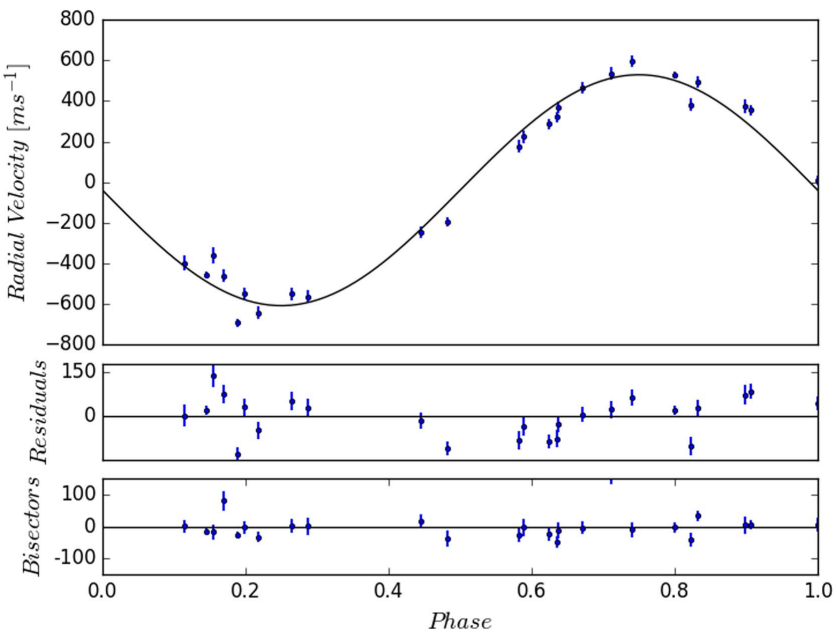

Figure 4. Orbital solution for Qatar-5b, showing the velocity curve and observed velocities.

Table 5

Initial Orbital Parameters

\begin{tabular}{llll}
\hline \hline Orbital Parameter & Qatar-3b & Qatar-4b & Qatar-5b \\
\hline Semi-amplitude $K\left(\mathrm{~m} \mathrm{~s}^{-1}\right)$ & $594 \pm 70$ & $1087 \pm 88$ & $570 \pm 17$ \\
Relative $\gamma\left(\mathrm{m} \mathrm{s}^{-1}\right)$ & $542 \pm 44$ & $927 \pm 74$ & $416 \pm 13$ \\
Absolute $\gamma\left(\mathrm{km} \mathrm{s}^{-1}\right)$ & $+6.04 \pm 0.1$ & $-28.76 \pm 0.1$ & $-9.54 \pm 0.1$ \\
rms RV residuals $\left(\mathrm{m} \mathrm{s}^{-1}\right)$ & 217 & 263 & 67 \\
Number of RVs & 27 & 10 & 25 \\
Reduced $\chi^{2} \mathrm{RV}$ & 7.1 & 6.9 & 4.5 \\
\hline
\end{tabular}

installed at the QES station hosted by the New Mexico Skies Observatory on the nights of August 17 and 2016 September 6. QFT is equipped with Andor iKon-M 934 deep depletion, back illuminated CCD camera optimized for follow-up photometric studies. Qatar-4b light curves were obtained through a Johnson-I filter, using a defocusing technique similar to our Calar Alto observations and an exposure time of $180 \mathrm{~s}$ per frame. A follow-up light curve for Qatar-5b was obtained using the KeplerCam on the $1.2 \mathrm{~m}$ telescope at the Fred L. Whipple Observatory on Mount Hopkins, Arizona on the night of 2015 November 10. KeplerCam is equipped with a single $4 \mathrm{~K} \times 4 \mathrm{~K}$ CCD covering an area of $23^{\prime} \times 23^{\prime}$ on the sky. The
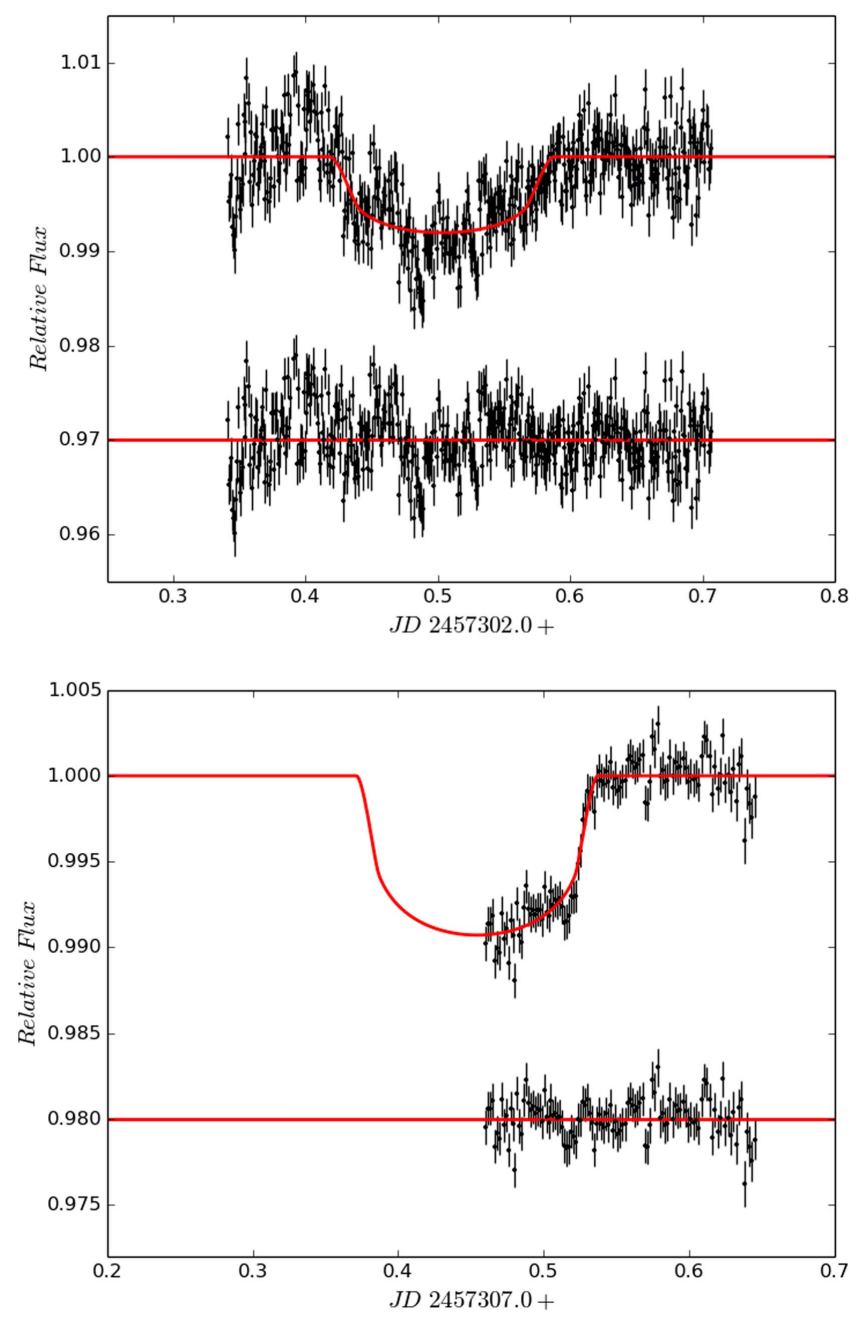

Figure 5. I-band follow-up light curves of Qatar-3b, obtained on 2015 October 06 (top panel) and 2015 October 11 (bottom panel) using the $1.23 \mathrm{~m}$ Zeiss telescope at the Calar Alto observatory. The best-fit transit model overlaid in red (see text for details). The light curves suffer from some extra noise due to the poor weather conditions.

observations were obtained through a SDSS- $i$ ' filter. Figures 57 show the follow-up light curves together with the model fits described in Section 3.2.

To better determine the transiting systems ephemerides, we fit the follow-up photometric curves with a transiting model following the prescription outlined in Pál (2008). In short, the Pál (2008) method uses analytical expressions to evaluate the partial derivatives of the flux decrease function for an eclipsed star, under the assumption of quadratic limb darkening. Pál (2008) equations allow for a clear separation between terms depending only on the limb darkening coefficients, and terms depending only on the occultation geometry.

After the model fit, we estimate the $T_{C}$ and calculate the best ephemerides. For the current ephemerides, we used the $T_{C}$ from the best model fit of the light curve. Ephemerides are listed in Table 6. Note that we follow the standard procedure and we did not include the discovery light curves in the physical parameter analysis. The discovery light curve data points have overly large error bars, so we used only the follow-up high precision light curves in order to reduce the errors in the physical parameters. 

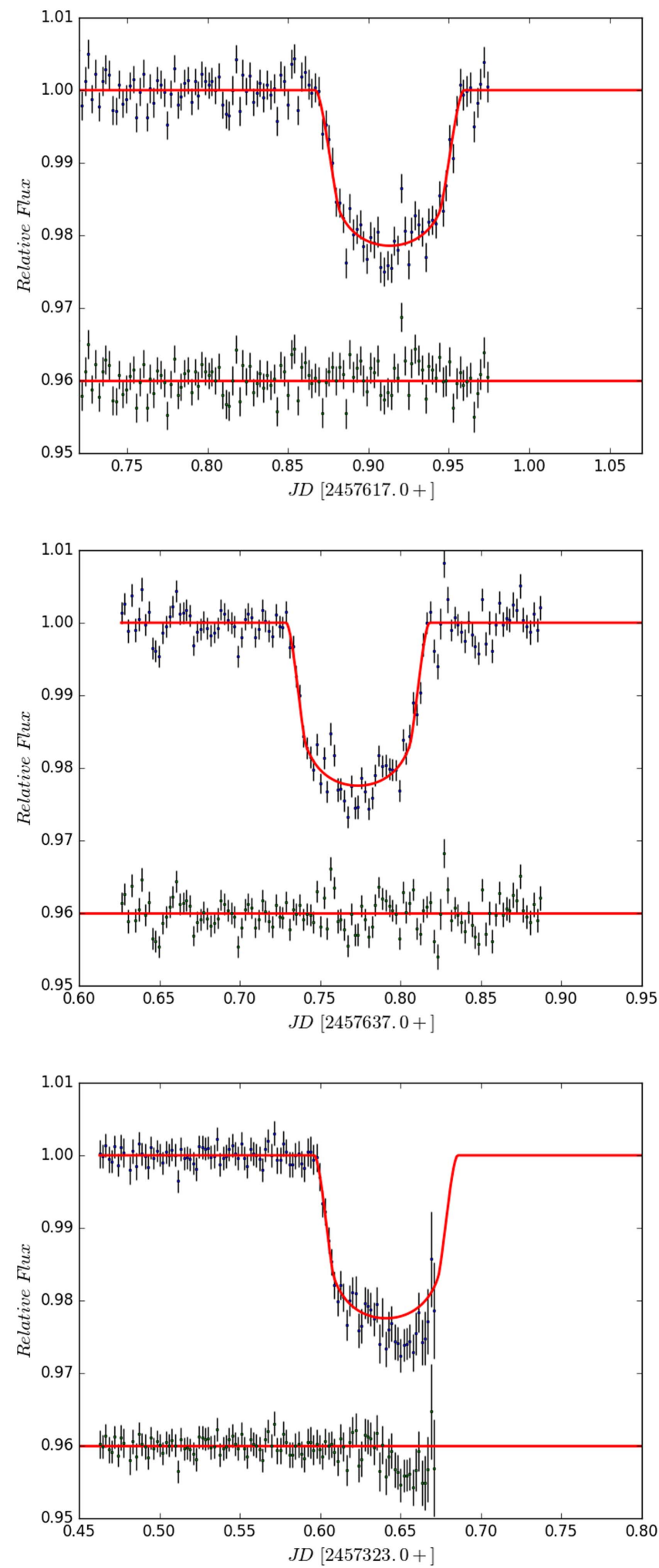

Figure 6. Same as Figure 5, but for Qatar-4b, observed on 2015 October 27 using the $1.23 \mathrm{~m}$ Zeiss telescope at the Calar Alto observatory, and on 2016 August 17 and 2016 September 06 using the QFT.

\section{Analysis and Results}

\subsection{Stellar Parameters}

To improve the characterization of the three host stars, we analyzed the TRES spectra using the Stellar Parameter

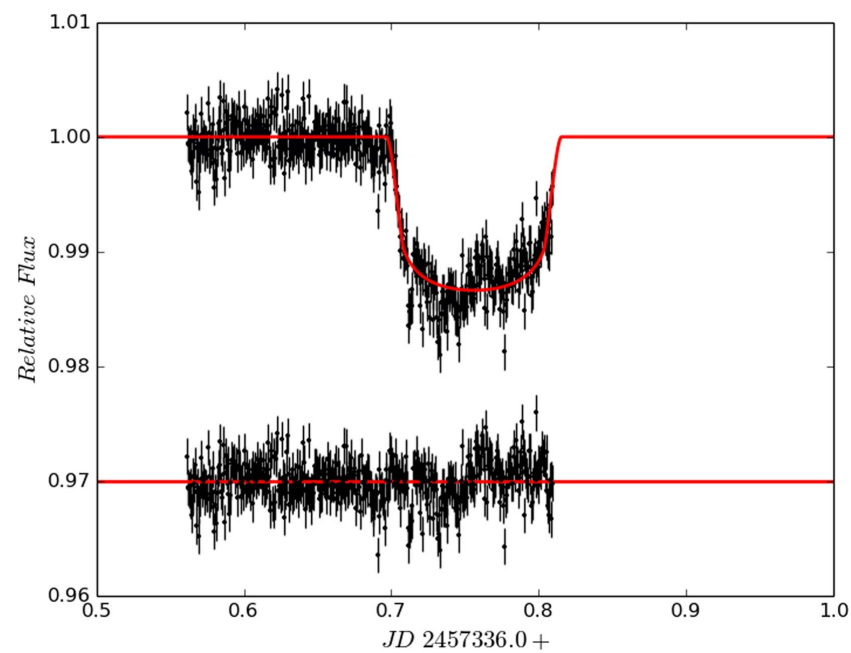

Figure 7. Same as Figure 5, but for Qatar-5b, observed on 2015 November 10 with KeplerCam.

Classification (SPC) tool developed by Buchhave et al. (2012). In brief, the SPC cross correlates the observed spectrum with a library of synthetic spectra from Kurucz model atmospheres and finds the stellar parameters from a multidimensional surface fit to the peak correlation values. We used the ATLAS9 grid of models with the new Opacity Distribution Functions from Castelli \& Kurucz (2004). In addition, the stellar parameters-effective temperature $\left(T_{\text {eff }}\right)$, metallicity $([m / H])$, surface gravity $(\log g)$, and projected rotational velocity $v \sin i$-for the hosts were derived from the co-added spectra of each star through spectral modelling using the Spectroscopy Made Easy (SME) package (Valenti \& Piskunov 1996).

We note that the values of $T_{\text {eff }}$, estimated via SPC and SME, are within $3.5 \sigma$ (for Qatar-3), $2 \sigma$ (Qatar-4), and $1 \sigma$ (Qatar-5) of each other, while the $\log g$ and $v \sin i$ values for all three stars are essentially the same (differences are less than $1 \sigma$ ). The only noticeable difference is metallicity $([\mathrm{m} / H])$, where SME gives systematically lower values relative to SPC by 0.3 . We examine the effect of these differences on the calculated planetary parameters (mass and radius) in the next section. In Table 6 we also provide the ages of the host stars using gyrochronology equations from Brown (2014)_Equation (1), assuming that the stellar rotation axis is perpendicular to the orbital plane. We found that the ages for all three stars are $\tau_{\mathrm{Gyr}, \mathrm{Q} 3}=0.31 \mathrm{Gyr}, \quad \tau_{\mathrm{Gyr}, \mathrm{Q} 4}=0.17 \mathrm{Gyr}, \quad$ and $\tau_{\mathrm{Gyr}, \mathrm{Q} 5}=0.53$ Gyr for Qatar-3b, Qatar-4b, and Qatar-5b, respectively. Additionally, using model isochrones from Dotter et al. (2008) and the input parameters for Table 6, we calculate independent values for the ages of the host stars $0.1<\tau_{\text {iso,Q3,Q4,Q5 }}<0.3 \mathrm{Gyr}$. All host stars are relatively young stars, which is basically consistent with their relatively fast rotation $\left(v \sin i>5 \mathrm{~km} \mathrm{~s}^{-1}\right)$. We note that previous studies (Brown 2014; Maxted et al. 2015) show that, in general, gyrochronology suggests a younger age than isochrone models. In our case the ages from both methods-gyrochronology and model isochrones - are generally consistent with each other.

\subsection{Planetary System Parameters}

To determine the physical parameters of the three planetary systems, we run a global solution of the available RV and transit photometric data using the EXOFAST package 
Table 6

Median Values and 68\% Confidence Intervals

\begin{tabular}{|c|c|c|c|c|}
\hline Parameter & Units & Qatar-3b & Qatar-4b & Qatar-5b \\
\hline \multicolumn{5}{|c|}{ Stellar Parameters: } \\
\hline$M_{*}$ & $\operatorname{Mass}\left(M_{\odot}\right)$ & $1.145 \pm 0.064$ & $0.896 \pm 0.048$ & $1.128 \pm 0.056$ \\
\hline$R_{*}$ & Radius $\left(R_{\odot}\right)$ & $1.272 \pm 0.14$ & $0.849 \pm 0.063$ & $1.076 \pm 0.051$ \\
\hline$L_{*}$ & Luminosity $\left(L_{\odot}\right)$ & $1.90 \pm 0.46$ & $0.481 \pm 0.076$ & $1.138 \pm 0.12$ \\
\hline$\rho_{*}$ & Density $\left(\mathrm{g} \mathrm{cm}^{-3}\right)$ & $0.78 \pm 0.20$ & $2.07 \pm 0.038$ & $1.286 \pm 0.15$ \\
\hline $\log \left(g_{*}\right)$ & Surface gravity (cgs) & $4.286 \pm 0.079$ & $4.533 \pm 0.058$ & $4.427 \pm 0.035$ \\
\hline$T_{\text {eff }}$ & Effective temperature $(\mathrm{K})$ & $6007 \pm 52$ & $5215 \pm 50$ & $5747 \pm 49$ \\
\hline$[\mathrm{Fe} / \mathrm{H}]$ & Metallicity & $-0.041 \pm 0.081$ & $0.103 \pm 0.081$ & $0.377 \pm 0.080$ \\
\hline Age & Age (Gyr) & $0.310 \pm 0.001$ & $0.170 \pm 0.010$ & $0.530 \pm 0.004$ \\
\hline$P_{\text {rot }}$ & Rotation period (days) & 6.31 & 6.05 & 12.10 \\
\hline \multicolumn{5}{|c|}{ Planetary Parameters: } \\
\hline$P$ & Period (days) & 2.5079204 & 1.8053564 & 2.8792319 \\
\hline$a$ & Semimajor axis (au) & $0.03783 \pm 0.00069$ & $0.02803 \pm 0.00048$ & $0.04127 \pm 0.00067$ \\
\hline$M_{\mathrm{P}}$ & Mass $\left(M_{\mathrm{J}}\right)$ & $4.31 \pm 0.47$ & $6.10 \pm 0.54$ & $4.32 \pm 0.18$ \\
\hline$R_{\mathrm{P}}$ & Radius $\left(R_{\mathrm{J}}\right)$ & $1.096 \pm 0.14$ & $1.135 \pm 0.11$ & $1.107 \pm 0.064$ \\
\hline$\rho_{\mathrm{P}}$ & Density $\left(\mathrm{g} \mathrm{cm}^{-3}\right)$ & $4.0 \pm 1.2$ & $5.10 \pm 1.00$ & $3.95 \pm 0.58$ \\
\hline $\log \left(g_{\mathrm{P}}\right)$ & Surface gravity & $3.942 \pm 0.10$ & $4.069 \pm 0.078$ & $3.940 \pm 0.044$ \\
\hline$T_{\mathrm{eq}}$ & Equilibrium temperature $(\mathrm{K})$ & $1681 \pm 84$ & $1385 \pm 48$ & $1415 \pm 31$ \\
\hline$\Theta$ & Safronov number & $0.256 \pm 0.035$ & $0.336 \pm 0.035$ & $0.284 \pm 0.016$ \\
\hline$\langle F\rangle$ & Incident flux $\left(10^{9} \mathrm{erg} \mathrm{s}^{-1} \mathrm{~cm}^{-2}\right)$ & $1.81 \pm 0.39$ & $0.835 \pm 0.12$ & $0.910 \pm 0.082$ \\
\hline$e$ & Eccentricity (fixed) & 0 & 0 & 0 \\
\hline \multicolumn{5}{|c|}{ RV Parameters: } \\
\hline$K$ & RV semi-amplitude $\left(\mathrm{m} \mathrm{s}^{-1}\right)$ & $587 \pm 58$ & $1087 \pm 88$ & $568 \pm 15$ \\
\hline$\gamma$ & Systemic velocity $\left(\mathrm{m} \mathrm{s}^{-1}\right)$ & $542 \pm 36$ & $1022 \pm 74$ & $416 \pm 11$ \\
\hline \multicolumn{5}{|c|}{ Primary Transit Parameters: } \\
\hline$T_{C}$ & Time of transit $\left(\mathrm{BJD}_{\mathrm{TDB}}\right)$ & $2457302.453004 \pm 0.00010$ & $2457637.77361 \pm 0.00046$ & $2457336.758242 \pm 0.000098$ \\
\hline$R_{\mathrm{P}} / R_{*}$ & Radius of planet in stellar radii & $0.0888 \pm 0.0018$ & $0.1379 \pm 0.0030$ & $0.1061 \pm 0.0013$ \\
\hline$a / R_{*}$ & Semimajor axis in stellar radii & $6.39 \pm 0.59$ & $7.11 \pm 0.48$ & $8.27 \pm 0.34$ \\
\hline$u_{1}$ & linear limb-darkening coeff & $0.258 \pm 0.047$ & $0.400 \pm 0.048$ & $0.323 \pm 0.043$ \\
\hline$u_{2}$ & quadratic limb-darkening coeff & $0.293 \pm 0.050$ & $0.233 \pm 0.050$ & $0.271 \pm 0.048$ \\
\hline$i$ & Inclination $\left({ }^{\circ}\right)$ & $86.8 \pm 2.0$ & $87.5 \pm 1.6$ & $88.74 \pm 0.87$ \\
\hline$b$ & Impact parameter & $0.35 \pm 0.22$ & $0.31 \pm 0.19$ & $0.18 \pm 0.16$ \\
\hline$\delta$ & Transit depth & $0.00789 \pm 0.00032$ & $0.01902 \pm 0.00083$ & $0.01126 \pm 0.00028$ \\
\hline$\tau$ & Ingress/egress duration (days) & $0.0120 \pm 0.0028$ & $0.0118 \pm 0.0022$ & $0.01201 \pm 0.0013$ \\
\hline$T_{14}$ & Total duration (days) & $0.1296 \pm 0.0034$ & $0.0891 \pm 0.0019$ & $0.1212 \pm 0.0015$ \\
\hline
\end{tabular}

Note. We assume $R_{\odot}=696342.0 \mathrm{~km}, M_{\odot}=1.98855 \times 10^{30} \mathrm{~kg}, R_{\mathrm{J}}=69911.0 \mathrm{~km}, M_{\mathrm{J}}=1.8986 \times 10^{27} \mathrm{~kg}$ and $1 \mathrm{au}=149597870.7 \mathrm{~km}$.

(Eastman et al. 2013). The transit light curves include only the follow-up photometric data and not the discovery light curves. As described by the authors, the EXOFAST performs a simultaneous fit of the RV and/or transit data for a single planet. In our case, for all the systems, we fixed the planetary orbital period to the value determined from the transits ephemerides and set the initial stellar parameters $\left(T_{\text {eff }}, \log g\right.$, $[\mathrm{Fe} / \mathrm{H}])$ to the values determined from the spectroscopic analysis of the host stars.

To quantify the effect different sets of values for $T_{\text {eff }}, \log g$, $[\mathrm{Fe} / \mathrm{H}]$, estimated via SPC and SME, have on the calculated values for the planetary mass and radius $\left(M_{\mathrm{P}}, R_{\mathrm{P}}\right)$, we fed EXOFAST with the sets of initial stellar parameters determined by SPC and SME separately and compared the results. We remind the reader that internally EXOFAST uses the Torres relations (Torres et al. 2010) — calibrations based on accurate $(\leqslant 3 \%)$ masses and radii from detached binary systems-to determine the masses and radii of the host stars. These relations are valid for main sequence stars above $0.6 M_{\odot}$, and we note that all our stars have estimated ages and masses well within the range covered by the Torres relations.

The nature of the Torres relations is such that $M_{*}$ and $R_{*}$ are only weakly dependent on metallicity. As a result, the SPC and
SME sets of values lead to very similar host stellar masses and radii-indistinguishable for Qatar-3; 4\%, and 5\% difference in $M_{*}$ and $R_{*}$, respectively for Qatar-4; and $4 \%$, and $2 \%$ difference in $M_{*}$ and $R_{*}$, respectively for Qatar-5. The biggest differences are in the luminosity of the host stars where the SME sets of parameters lead to a $13 \%$ more luminous star for Qatar-3, a 15\% more luminous star for Qatar-4, but essentially the same for Qatar-5.

Most importantly, differences in stellar parameters produced via SPC and SME lead to insignificant differences in the derived values for the planetary masses and radii. In all three cases-Qatar-3b, Qatar-4b, and Qatar-5b-these are well within $1 \sigma$ of the uncertainty. For this reason, in all tables, we list only the values derived with the initial set of stellar parameters determined via SPC.

The initial evaluation of the fits to the RV curves indicated that they were all well described by circular orbits, i.e., $e=0$. This is not surprising, as all three planets have short period orbits that are expected to have circularized. In addition, in the case of Qatar-4b, the RV curve has relatively few points and does not warrant a detailed search for an eccentric solution. In the cases of Qatar-3b and Qatar-5b we searched for eccentric solutions as well, but the results were essentially 
indistinguishable from $e=0$ at the $\leqslant 2 \sigma$ level. Consequently, in our global fits we kept the eccentricity fixed at $e=0$. In addition, the period of each planet was kept fixed at the value determined by the transit ephemerides by in practice allowing it to vary only at the insignificant $10^{-5}$ days level.

Table 6 summarises the physical parameters of the planets. The best fit for both RV and photometric light curves comes from EXOFAST. The Safronov numbers for each planet are not used in the current paper and are provided in Table 6 for completeness, as they may be useful for other studies.

\section{Discussion}

Using the equations from Leconte et al. (2010) and Jackson et al. (2008), we calculate the tidal interaction timescale for the eccentricity evolution of the systems. We used the values from Table 6 of $M_{\star}, R_{\star}, M_{\mathrm{P}}, R_{\mathrm{P}}$, assuming tidal quality factors of $Q_{\star}=10^{6.5}$ and $Q_{\mathrm{P}}=10^{5.5}$. The three rotation periods given in Table $6-P_{\mathrm{Q} 3}=6.31$ days, $P_{\mathrm{Q} 4}=6.05$ and $P_{\mathrm{Q} 5}=12.10$ days, respectively - are calculated using the stellar radii from our solutions, the $v \sin i$ form our spectra, and assuming the stellar rotation axis and the planet orbit are coplanar. Finally the timescales for the eccentric evolution is $\tau_{\mathrm{Q} 3}=0.133 \mathrm{Gyr}$, $\tau_{\mathrm{Q} 4}=0.0870 \mathrm{Gyr}, \tau_{\mathrm{Q} 3}=0.544 \mathrm{Gyr}$ for the three systems, respectively. The eccentricity evolution timescale for Qatar$5 \mathrm{~b}$, is approximately equal with its age (Table 6).

The three planets presented here fall in the area of heavy hot Jupiters with masses in the range $4-6 M_{\mathrm{J}}$ and densities $4-5 \mathrm{~g} \mathrm{~cm}^{-3}$ (see Table 6). Their equilibrium temperatures place them in the pL class of planets following the Fortney et al. (2008) nomenclature. To put the properties of the three new planets in perspective we show their positions on the planet mass-radius (Figure 8, top) and mass-density (Figure 8, bottom) diagrams and compare them with data for the well studied transiting exoplanets from TEPcat. ${ }^{8}$ On both the massradius and mass-density diagrams, the three new planets occupy the sparsely populated area of relatively heavy and dense planets on one end of the parameter space. Qatar $3 b$ and Qatar $5 b$ have very similar characteristics and project almost on top of each other on each panel of Figure 8 and close to the theoretical models and in company of some other observed planets. Qatar 4b, on the other hand, appears somewhat bloated compared with other planets with similar masses and occupies a place on the edge of the parameter space though not very far from previously observed planets. On the mass-density diagram we also show the $0.3 \mathrm{Gyr}$ model isochrones from Fortney et al. (2007) ${ }^{9}$ for giant planets with different core mass values at a distance of $0.045 \mathrm{au}$. The three planes occupy the area of the mass-density diagram which is insensitive to a particular core mass value.

\section{Conclusions}

Qatar-3b, Qatar-4b, and Qatar-5b are three new transiting hot Jupiters hosted by K1V, G0V, and G2V stars respectively. All three are short period planets $\left(P_{\mathrm{Q} 3 \mathrm{~b}}=2.50792, P_{\mathrm{Q} 4 \mathrm{~b}}=\right.$ 1.80539 , and $P_{\mathrm{Q} 5 \mathrm{~b}}=2.87923$ days) with with masses and radii $\quad\left(M_{\mathrm{Q} 3 \mathrm{~b}}=4.31 M_{\mathrm{J}} \quad R_{\mathrm{Q} 3 \mathrm{~b}}=1.096 R_{\mathrm{J}}, \quad M_{\mathrm{Q} 4 \mathrm{~b}}=6.10 M_{\mathrm{J}}\right.$ $\left.R_{\mathrm{Q} 4 \mathrm{~b}}=1.135 R_{\mathrm{J}}, \quad M_{\mathrm{Q} 5 \mathrm{~b}}=4.32 M_{\mathrm{J}} \quad R_{\mathrm{Q} 5 \mathrm{~b}}=1.107 R_{\mathrm{J}}\right)$ in the expected regime for hot Jupiters, and densities ranging between

\footnotetext{
8 The Transiting Extrasolar Planet Catalog (TEPcat) is available at http:// www.astro.keele.ac.uk/jkt/tepcat/.

9 Models are available at http://www.ucolick.org/ jfortney/models.htm.
}
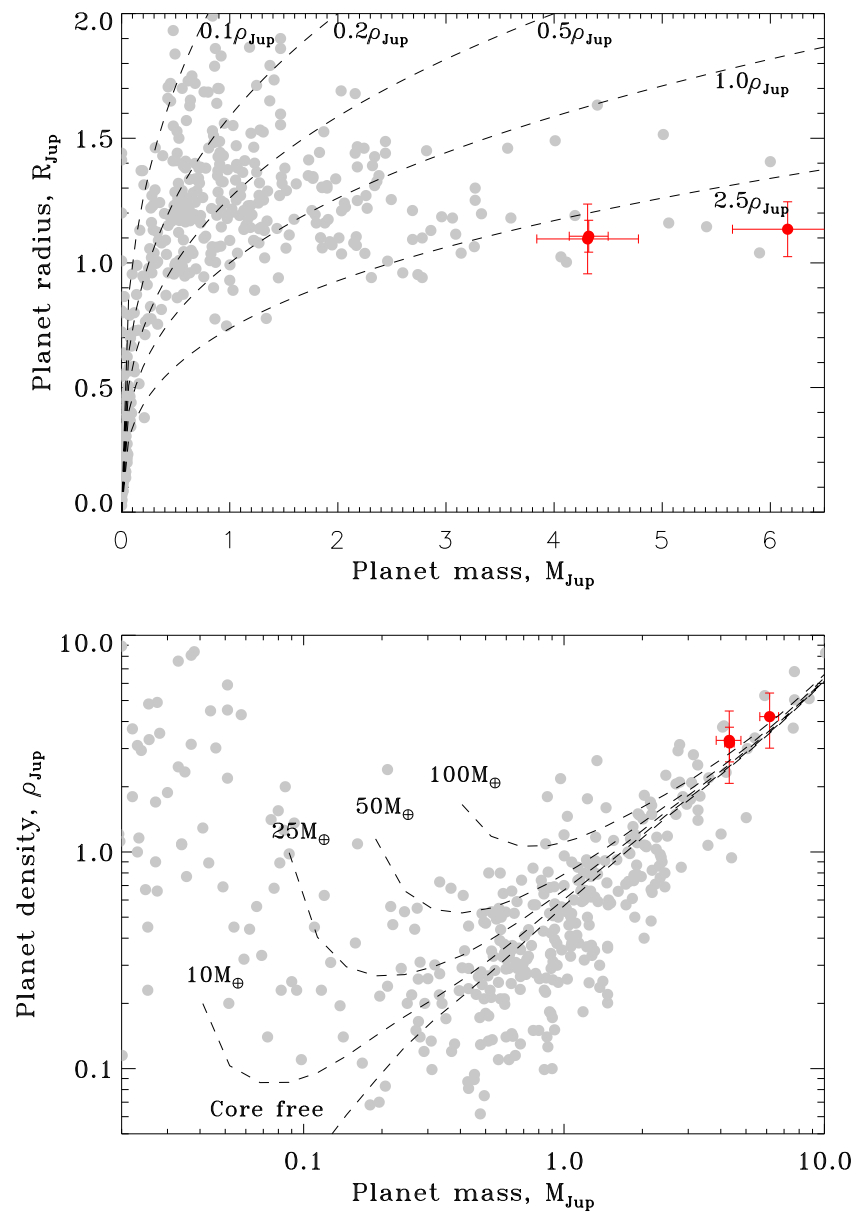

Figure 8. Top: mass-radius diagram of known transiting exoplanets. Light gray points represent data for well-studied planets from the TEPcat. Error bars are supfpressed for clarity. Qatar-3b, Qatar- $4 \mathrm{~b}$ and Qatar-5b are shown as red points with error bars. Dashed lines show constant density for $0.1,0.25,0.5$, 1.0, and $2.5 \rho_{\text {Jup }}$. Bottom: the mass-density diagram for currently known exoplanets (also taken form TEPcat). As in the previous panel the new planets are shown as red dots with error bars. Models for giant planets with different core masses are drawn as dashed lines for comparison.

2 and $4 \mathrm{~g} \mathrm{~cm}^{-3}$. The planets look similar to other members of the hot Jupiter family on the mass-radius and mass-density diagrams. We note, however, that all three planets reside in the sparsely populated heavy-mass end $\left(M>4 M_{\mathrm{J}}\right)$ on the massradius diagram. In addition, Qatar-4b appears significantly bloated for its mass. Future follow-up observations will help characterize these planets in greater detail and help shed light on some of the peculiarities.

This publication is supported by NPRP grant no. X-019-1006 from the Qatar National Research Fund (a member of Qatar Foundation). The statements made herein are solely the responsibility of the authors. D.F.E. is funded by the UK Science and Technology Facilities Council.

\section{References}

Alsubai, K. A., Parley, N. R., Bramich, D. M., et al. 2013, AcA, 63, 465 Barnes, S. 2007, ApJ, 669, 1167

Batalha, N. M., Rowe, J. F., Bryson, S. T., et al. 2013, ApJS, 204, 24

Bramich, D. M. 2008, MNRAS, 386, L77

Brown, D. J. A. 2014, MNRAS, 442, 1844

Buchhave, L. A., Bakos, G., Hartman, J. D., et al. 2010, ApJ, 720, 1118

Buchhave, L. A., Latham, D., Johansen, A., et al. 2012, Natur, 486, 375 
Castelli, F., \& Kurucz, R. L. 2004, astro-ph/0405087

Collier Cameron, A., Pollacco, D., Street, R. A., et al. 2006, MNRAS, 373, 799C

Dotter, A., Chaboyer, B., Jevremović, D., et al. 2008, ApJS, 178, 89

Eastman, J., Gaudi, B. S., \& Agol, E. 2013, PASP, 125, 83

Fortney, J. J., Lodders, K., Marley, M. S., \& Freedman, R. S. 2008, ApJ, 678,1419

Fortney, J. J., Marley, M. S., \& Barnes, J. W. 2007, ApJ, 659, 1661

Jackson, B., Greenberg, R., \& Barnes, R. 2008, ApJ, 678, 1396J

Kass, R., \& Raftery, A. 1995, J. Am. Stat. Assoc., 90, 773

Kovács, G., Bakos, G., \& Noyes, R. 2005, MNRAS, 356, 557

Kovács, G., Zucker, S., \& Mazeh, T. 2002, A\&A, 391, 369
Leconte, J., Chabrier, G., Baraffe, I., \& Levrard, B. 2010, A\&A, 516, 64

Lucy, L. B., \& Sweeney, M. A. 1971, ApJ, 76, 544

Maxted, P. F. L., Serenelli, A. M., \& Sputhworth, J. 2015, A\&A, 577, 90

Pál, A. 2008, MNRAS, 390, 281

Rawlings, J. O., Pantula, S. G., \& Dickey, D. A. 1998, Applied Regression Analysis: A Research Tool (2nd ed.; New York: Springer)

Southworth, J., Hinse, T. C., Jørgensen, U. G., et al. 2009, MNRAS, 396, 1023

Southworth, J., Hinse, T. C., Burgdorf, M., et al. 2014, MNRAS, 444, 776

Tamuz, O., Mazeh, T., \& Zucker, S. 2005, MNRAS, 356, 1466

Torres, G., Andersen, J., \& Giménez, A. 2010, A\&ARv, 18, 67

Valenti, J. A., \& Piskunov, N. 1996, A\&AS, 118, 595 